\title{
A REMARK ON COINCIDENCE THEOREMS
}

\author{
E. TARAFDAR AND XIAN-ZHI YUAN
}

(Communicated by Palle E. T. Jorgensen)

\begin{abstract}
Using a different approach, we prove a general coincidence theorem of multivalued mappings which have contractible values in non-convex setting.
\end{abstract}

Let $\mathbb{N}$ and $\mathbb{R}$ denote the set of all natural numbers and the set of all real numbers, respectively. For each $n \in \mathbb{N}$, let $N=\{0,1, \cdots, n\}$ and $\Delta_{N}=$ $\operatorname{co}\left\{e_{0}, \cdots, e_{n}\right\}$ be the standard simplex of dimension $n$, where $\left\{e_{0}, \cdots, e_{n}\right\}$ is the canonical basis of $\mathbb{R}^{n+1}$. For $J \in \mathscr{F}(N), \Delta_{J}=\operatorname{co}\left\{e_{j}: j \in J\right\}$, where $\mathscr{F}(N)$ denotes the family of all non-empty subsets of $N$. A topological space $X$ is said to be contractible if the identity mapping $I_{X}$ of $X$ is homotopic to a constant function. Given a non-empty set $X$, let $2^{X}$ denote the family of all non-empty subsets of $X$.

In 1986, by using Browder's selection theorem (e.g., see [2]) for the multivalued mapping with open inverse values, Komiya [10] gave the following coincidence theorem:

Theorem (Komiya). Let $X$ be a non-empty convex subset of a Hausdorff topological vector space $E$, and let $Y$ be a non-empty compact convex subset of a Hausdorff topological vector space $W$. Suppose $A: X \rightarrow 2^{Y}$ is upper semicontinuous with closed and convex values and $B: Y \rightarrow 2^{X}$ has convex values such that $B^{-1}(x)$ is open in $Y$ for each $x \in X$. Then there exists $\left(x_{0}, y_{0}\right) \in X \times Y$ such that $x_{0} \in B\left(y_{0}\right)$ and $y_{0} \in A\left(x_{0}\right)$.

In this note, we shall give a very general form of the above coincidence theorem in a non-convex setting by using a different but simple approach.

The following is Theorem 1 of Horvath [7] (see also Theorem 1 of [8]):

Lemma 1. Let $X$ be a topological space. For each non-empty subset $J$ of $N=$ $\{0,1, \cdots, n\}$, let $F_{J}$ be a non-empty contractible subset of $X$ with $F_{J} \subset$ $F_{J^{\prime}}$ whenever $\varnothing \neq J \subset J^{\prime} \subset\{0,1, \cdots, n\}$. Then there exists a continuous function $f: \Delta_{N} \rightarrow X$ such that $f\left(\Delta_{J}\right) \subset F_{J}$ for each non-empty subset $J$ of $\{0,1, \cdots, n\}$.

By employing the same arguments of Eilenberg and Montgomery [3, pp. 216217] and using Theorem 6.3 of Gòrniewicz [4, p. 111] instead of the coincidence

Received by the editors January 27, 1994.

1991 Mathematics Subject Classification. Primary 47H10, 49A40.

Key words and phrases. Contractible, coincidence theorem, upper semicontinuous. 
theorem used in [3, pp. 216-217], the following lemma was obtained by Shioji $[11$, p. 188]:

Lemma 2 (Eilenberg and Montgomery, Gòrniewicz, and Shioji). Let $\Delta_{N}$ be an n-dimensional simplex with the Euclidean topology, and let $Y$ be a compact topological space. Let $\psi: Y \rightarrow \Delta_{N}$ be a single-valued continuous map, and let $T: \Delta_{N} \rightarrow 2^{Y}$ be a set-valued upper semicontinuous mapping with non-empty compact contractible values. Then there exists $x_{0} \in \Delta_{N}$ such that $x_{0} \in \psi \circ T\left(x_{0}\right)$, where $\psi \circ T$ denotes the composition of mapping $T$ with $\psi$.

Now we have the following:

Theorem 1. Let $X$ be a contractible space and $Y$ a Hausdorff compact space. Let $A: X \rightarrow 2^{Y}$ be upper semicontinuous with non-empty compact contractible values. Suppose that $B: Y \rightarrow 2^{X}$ is such that

(a) $B^{-1}(x)$ is open for each $x \in X$ and

(b) for each open set $S$ in $Y$, the set $\cap_{y \in S} B(y)$ is empty or contractible.

Then there exist $w_{0} \in X$ and $z_{0} \in Y$ such that $w_{0} \in B\left(z_{0}\right)$ and $z_{0} \in A\left(w_{0}\right)$. Proof. We first show that there exist an $n$-simplex $\Delta_{N}$ and two functions $f$ : $\Delta_{N} \rightarrow X$ and $\psi: Y \rightarrow \Delta_{N}$ such that $f(\psi(y)) \in B(y)$ for all $y \in Y$.

Since $Y$ is compact, by (a), there exists a finite subset $\left\{x_{0}, \cdots, x_{n}\right\}$ of $X$ such that $Y=\cup_{i=0}^{n} B^{-1}\left(x_{i}\right)$. Now for each non-empty subset $J$ of $N:=$ $\{0, \cdots, n\}$, we define

$$
F_{J}= \begin{cases}\cap\left\{B(y): y \in \cap_{j \in J} B^{-1}\left(x_{j}\right)\right\}, & \text { if } \cap_{j \in J} B^{-1}\left(x_{j}\right) \neq \varnothing, \\ X, & \text { otherwise. }\end{cases}
$$

Note that if $y \in \cap_{j \in J} B^{-1}\left(x_{j}\right)$, then $\left\{x_{j}: j \in J\right\} \subset B(y)$. If $\cap_{j \in J} B^{-1}\left(x_{j}\right) \neq \varnothing$, then $F_{J}=\cap\left\{B(y): y \in \cap_{j \in J} B^{-1}\left(x_{j}\right)\right\}$ is non-empty and contractible. It is clear that $F_{J} \subset F_{J^{\prime}}$ whenever $\varnothing \neq J \subset J^{\prime} \subset\{0, \cdots, n\}$. Thus $F$ satisfies all hypotheses of Lemma 1. By Lemma 1, there is a continuous function $f$ : $\Delta_{N} \rightarrow X$ such that $f\left(\Delta_{J}\right) \subset F_{J}$ for all $J \in \mathscr{F}(N)$. Let $\left\{\psi_{i}: i \in N\right\}$ be a continuous partition of unity subordinated to the covering $\left\{B^{-1}\left(x_{i}\right): i \in\right.$ $N\}$, i.e., for each $i \in N, \psi_{i}: Y \rightarrow[0,1]$ is continuous, $\left\{y \in Y: \psi_{i}(y) \neq\right.$ 0\} $\subset B^{-1}\left(x_{i}\right)$ such that $\sum_{i=0}^{n} \psi_{i}(y)=1$ for all $y \in Y$. Define $\psi: Y \rightarrow$ $\Delta_{N}$ by $\psi(y)=\left(\psi_{0}(y), \psi_{1}(y), \cdots, \psi_{n}(y)\right)$ for each $y \in Y$. Then $\psi(y) \in$ $\Delta_{J(y)}$ for all $y \in Y$, where $J(y)=\left\{i \in\{0, \cdots, n\}: \psi_{i}(y) \neq 0\right\}$. Therefore $f(\psi(y)) \in f\left(\Delta_{J(y)}\right) \subset F_{J(y)} \subset B(y)$. Since $A$ is upper semicontinuous with nonempty compact and contractible values and $f$ is continuous, it follows that the composition $A \circ f: \Delta_{N} \rightarrow 2^{Y}$ is also upper semicontinuous with non-empty compact and contractible values. Note that $\psi: Y \rightarrow \Delta_{N}$ is continuous. By Lemma 2 above, there exists $x_{0} \in \Delta_{N}$ such that $x_{0} \in \psi \circ\left(A \circ f\left(x_{0}\right)\right)$. Let $w_{0}:=f\left(x_{0}\right)$. Then $w_{0}=f\left(x_{0}\right) \in f \circ\left(\psi \circ\left(A \circ f\left(x_{0}\right)\right)\right)=f \circ\left(\psi \circ\left(A\left(w_{0}\right)\right)\right)$ so that there exists $z_{0} \in A\left(w_{0}\right)$ such that $w_{0}=f \circ \psi\left(z_{0}\right) \in B\left(z_{0}\right)$.

Since each convex set in Hausdorff topological vector spaces is contractible, clearly Komiya's theorem is a special case of Theorem 1. Recently, by the generalization of the classic Knaster-Kuratowski-Mazurkiewicz theorem, Horvath [7] gave a number of coincidence theorems in which both mappings involve the property of open inverse values (or open image values); for example, see Theorem 3 and Corollaries 3-6 in [7]. Note that the mapping $A$ in Theorem 1 is upper semicontinuous, so that our Theorem 1 is not comparable with 
those coincidence results given by Horvath [7]. For more applications of coincidence theorems, we refer to Aubin [1], Browder [2], Granas and Liu [5], Ha [6], Komiya [10], Yuan [12], and the references therein.

\section{ACKNOWLEDGMENT}

The authors thank the referees for their comments in improving this note.

\section{REFERENCES}

1. J. P. Aubin, Mathematical methods of game and economic theory, revised version, NorthHolland, Amsterdam, 1982.

2. F. E. Browder, Coincidence theorems, minimax theorems and variational inequalities, Contemp. Math., vol. 26, Amer. Math. Soc., Providence, RI, 1984, pp. 67-80.

3. S. Eilenberg and D. Montgomery, Fixed point theorems for multi-valued transformations, Amer. J. Math. 68 (1946), 214-222.

4. L. Gòrniewicz, A Lefschetz-type fixed point theorem, Fund. Math. 88 (1975), 103-115.

5. A. Granas and F. C. Liu, Coincidences or set-valued maps and minimax inequalities, J. Math. Pures Appl. 65 (1986), 119-148.

6. C. W. Ha, Minimax and fixed point theorems, Math. Ann. 248 (1980), 73-77.

7. C. Horvath, Convexite generalisee et applications, Methodes Topologiques en Analyse Convexe: Partie des Comptes Rendus du Cours d'été OTAN “ Variational Method in Nonlinear Problems” (A. Granas, ed.), Presses Univ. Montréal, Montréal, Québec, Canada, 1990, pp. 79-99.

8. C. Horvath, Contractibility and generalized convexity, J. Math. Anal. Appl. 156 (1991), 341-357.

9. S. Kakutani, A generalization of Brouwer's fixed point theorem, Duke Math. J. 8 (1941), 457-459.

10. H. Komiya, Coincidence theorem and saddle point theorem, Proc. Amer. Math. Soc. 96 (1986), 599-602.

11. N. Shioji, A further generalization of the Knaster-Kuratowski-Mazurkiewicz theorem, Proc. Amer. Math. Soc. 111 (1991), 187-195.

12. X. Z. Yuan, Contributions to nonlinear analysis, Doctoral Thesis, Dalhousie University, Canada, 1993.

Department of Mathematics, The University of Queensland, Brisbane 4072, QueensLAND, AUSTRALIA

E-mail address: xzy@axiom.maths.uq.oz.au and eut@axiom.maths.uq.oz.au 